\title{
Discussion on the Influence of Cloud Computing to Library Management and Its
}

\section{Countermeasures}

\author{
Shan-shan $\operatorname{Han}^{1}$ \\ ${ }^{1}$ Library \\ Hebei University of Science and Technology \\ Shijiazhuang,China
}

\author{
Chi Han ${ }^{2}$ \\ ${ }^{2}$ Department of Pharmaceutical Engineering \\ The Chemical\&pharmaceutical College \\ Shijiazhuang,China
}

\begin{abstract}
Libraries have implemented cloud computing related network technology and services. Cloud computing will bring both challenges and benefits to library management The Challenges faced by library management includes alternative question, standard, data security and intellectual property. Librarianship should concern for: the principle of cloud computing; viability of library application; policy, standard and protocol of cloud computing; library administration based on cloud computing service; and case studies.
\end{abstract}

Keywords: Cloud computing; Library management; Influence;Countermeasures

\section{INTRODUCTION}

Cloud computing concept is one of the most exciting events in the field of computing in 2008. The Pugh Internet and United States life project announced a survey, although many users may not be familiar with the word, but $69 \%$ of users have been using cloud computing. Thus, cloud computing has been not just the hype of large network companies, but a real service mode.

From the view of library automation development history, almost every new information technology appearances can cause the library community to pay great attention and promote the library management and service upgrade. Due to the emerging cloud computing services is expected from the infrastructure level solve many long plagued the library network information management and service existing problem, as might be expected, will have more and more libraries trying to apply cloud computing.
The cloud computing can improve the level of management and service of library network information and reduce the cost of management and service ${ }^{[1]}$.

From the basic concept of cloud computing and the possible framework of library application cloud computing, the challenges to library management brought by cloud computing is unprecedented. This requires the library industry to quickly respond to these challenges, and make a system and in-depth research on the application problem of cloud computing.

\section{Cloud computing and library}

Matrix once summed up the 20 definitions of cloud computing, but it is still far from covering the existing definitions. The chaos concept is one of the reasons of the library hostage doubting cloud computing. For the librarians, it should be appropriate to discuss the concept of cloud computing to keep a certain distance, and put the focus of theoretical attention on the application of cloud computing.

Before the emergence of the concept of cloud computing, there have been many computing network technology or service related to the cloud computing applied to the library. For example, bibliographic database and full-text database providers used a network database to replace the $\mathrm{CD}$ version many years ago. And more and more early mirror versions are replaced by the network version. In fact, the essence of the network version is connect the library to the cloud database. Software providers constantly try to change provision model of the software, the simply selling software products is replaced by the provision of IT services, such as the library 
automation management system hosting platform. The management software is installed in the vendor's server, and maintained by the manufacturer. The library realizes the automatic management of the business through a web browser. This software is the product of service mainly for small and medium libraries, or limited to some of the library's auxiliary applications, such as Feat Express Web to provide managed cross library retrieval system ${ }^{[2]}$.

\section{The influence of cloud computing to the library management}

In the application of cloud computing services, the libraries are liking other industries, face a variety of management issues. Combing the the research on cloud computing management in recent years and the application of the library, the main problem of library management are shown as follows:

\section{(1) Alternative problem}

The IT resources needed for the application of cloud management library service of the library in the ideal condition is that, there are some competing large cloud providers, providing the hardware, software, systems, platforms or infrastructure services for the library in the detachable way. That is to say, the library can select the most appropriate application from different cloud services, and is not entirely dependent on a service provider. The library should be free to choose the IT supplier, and transfer from one supplier to another with zero cost, or choose different services from different suppliers. Only in this case, the cloud computing is acceptable by the library. If the IT resource do not have the alternative feature, or is not free from a service provider transferred to another, or the transfer cost is very high, then the dependence of the library on cloud will become very strong, which is equivalent to the cloud provider binding. So the consequences is unbearable to contemplate ${ }^{[3]}$.

(2) Standard problem

To be able to achieve the alternative between different IT service providers, in addition to the corresponding agreement with suppliers through the formation of a contractual relationship, technically speaking, also need IT service provider's cloud computing can follow a common standard. Without the common standards, it is impossible to realize zero cost transferring from one supplier to another supplier. Due to the rapid development of cloud computing, big companies have "rushing", so the standard problem is more prominent. In the rapidly development of IT field, it is unrealistic to expect the national standards to be developed.. The current cloud computing standards, or as a result of the high market share of Microsoft products and natural standards, or industry organizations or a number of companies jointly developed standards.

(3) Data security and confidentiality problem

Data security is essential to the library. Both bibliographic data, reader data or circulation data, once lost, the consequences are be unbearable to contemplate. And the collection of digital data, once the library lost, it will be more heartache. But in fact, the hardware conditions and management ability of large computing center are better than those of ordinary library. In terms of technology, it is not less safer to set the data on the cloud than on the local hard disk. So the data security and confidentiality problem is more about the policy level. The management policies and corporate reputation of the cloud computing enterprise and even national policies, are likely to have a tremendous impact on cloud computing security.

(4) Intellectual property problem

After the user buying cloud computing, the own content will transfer to the cloud, and cloud computing companies host these elements. In theory, the user should have the right to own the managed data. That is, users want to be able to manage and control these contents, have the right to modify and delete these elements, and other people can not use and modify these elements. But in reality, cloud computing firm knows "data core" principles, so that they will do everything possible to make use of these data. And use the name of data integration, data mining and knowledge service to make the using way legal. The intellectual property rights arising from cloud computing may exceed the traditional intellectual property law category. Therefore, some scholars have proposed that the unique problem of cloud computing may indicate that the 
technology has reached the urgent need to reconsider the intellectual property license.

\section{The countermeasures to the influences}

Through the analysis of cloud computing in library application, we think that the focus of library science theory is the management problem caused by cloud computing, including:

(1) The basic theoretical issues of cloud computing

Research on information technology and its application have become the subject of the theories and methods of modern library science. Cloud computing is one of the new areas and new trend of information technology, and it may have a significant impact on the information technology application in the library. Cloud computing theory issues include the concept of cloud computing, the origin, basic concepts, types, features, and the difference between existing computing models, and so on. Although the research object is not cloud computing in the library or libraries for management application, but it can promote or deepen understanding of the library community for cloud computing applications, which can provide necessary policy thought and theory preparation for the library to deal with the cloud computing management challenges.

(2) The feasibility of cloud computing in the application of the library

Although the librarians keep an exploring attitude in the application of information technology, but library cloud computing applications, the system will be applied and all kinds of data placed in "the cloud" rather than stored locally, and librarians believing in "owns" concept will have a conflict. Therefore, the research on the feasibility of cloud computing application is necessary. Combining with cloud computing applied in libraries and other information management, explore the possible application of the field, study the cloud computing for the library automatic management system, database and application software selection.

(3) The policy, standards and agreements of the library cloud computing

Through the research of cloud computing related policies, contributed to the introduction of the related management regulations and industry standards or policies to ensure will be the implementation of cloud computing can meet the basic requirements of library management and service. Although cloud computing has been concerned about standards and protocols, but the library for cloud computing should also have industry standards. Library industry managers should organize the application of cloud computing standards and related agreements to study the formation of the industry's application code $\mathrm{e}^{[4]}$.

(4) The library management system based on cloud computing services

The library applications of cloud computing is a strategic choice, especially in the field of IT infrastructure. If the library once chooses the cloud computing, it is necessary to redeploy the management and service of the original information system, which will result in the changes of IT management system, even the entire changes of library business process. Therefore, it is necessary to study of cloud computing library mechanism and process of the IT infrastructure changes may leading to the changes, IT department staff number and structure changes, library for cloud calculation service quality detection and control means, and so on.

\section{Conclusions}

Through the basic concept of cloud computing and the possible framework of library application cloud computing, the challenges to library management brought by cloud computing is unprecedented. Librarianship need to concern about the principle of cloud computing, viability of library application, policy, standard and protocol of cloud computing, library administration based on cloud computing service and case studies. 


\section{References}

[1] Marcos D. Assunção, Rodrigo N. Calheiros, Silvia Bianchi, Marco A.S. Netto, Rajkumar Buyya, "Big Data computing and clouds: Trends and future directions," Journal of Parallel and Distributed Computing, pp. 3-15, 2015.

[2] Fan Bingsi, "Cloud Computing and Libraries Defense for Research on the Cloud Computing," Library and
Information Service, pp. 5-9, 2009.

[3] Jiang Liyan, "Cloud-based Information Platform Library," Journal of Modern Information, pp. 47-51, 2011.

[4] Chen Mang, Zhou Liqing, Zheng Cong, The Research on Construction and Performance of Library's Cheap Private Cloud Storage, Library Journal, pp. 85-93, 2015. 